\title{
Effects of COVID-19 Home Confinement on Mental Health in Individuals with Increased Risk of Alzheimer's Disease
}

\author{
Natalia Soldevila-Domenech ${ }^{\mathrm{a}, \mathrm{b}}$, Laura Forcano $^{\mathrm{a}, \mathrm{c}}$, Anna Boronat $^{\mathrm{a}}$, Thais Lorenzo ${ }^{\mathrm{a}, \mathrm{b}}$, Iris Piera $^{\mathrm{a}}$, \\ Albert Puig-Pijoan ${ }^{\mathrm{a}, \mathrm{d}}$, Julian Mateus ${ }^{\mathrm{a}}$, José María González de Echevarri Gómez ${ }^{\mathrm{e}, \mathrm{f}}$, Iva Knezevic ${ }^{\mathrm{e}}$, \\ Anna Soteras $^{\mathrm{e}}$, Karine Fauria ${ }^{\mathrm{e}, \mathrm{g}}$, Nieves Pizarro ${ }^{\mathrm{a}, \mathrm{b}}$, Jose Luis Molinuevo \\ and Rafael de la Torre ${ }^{\mathrm{a}, \mathrm{b}, \mathrm{c}, *}$ PENSA Study Group ${ }^{1}$ \\ a Integrative Pharmacology and Systems Neurosciences Research Group, Neurosciences Research Program, \\ Hospital del Mar Medical Research Institute (IMIM), Barcelona, Spain \\ ${ }^{\mathrm{b}}$ Department of Experimental and Health Sciences, University Pompeu Fabra, Barcelona, Spain \\ ${ }^{\mathrm{c} C I B E R}$ de Fisiopatología de la Obesidad y la Nutrición (CIBEROBN), Instituto de Salud Carlos III, Madrid, \\ Spain \\ ${ }^{\mathrm{d}}$ Cognitive Impairment and Movement Disorders Unit, Neurology Service, Hospital del Mar, Barcelona, Spain \\ ${ }^{\mathrm{e}}$ Barcelonaßeta Brain Research Center (BBRC), Pasqual Maragall Foundation, Barcelona, Spain \\ ${ }^{\mathrm{f}}$ Physiology of Cognition and Alzheimer's Prevention Research Group, Neurosciences Research Program, \\ Hospital del Mar Medical Research Institute (IMIM), Barcelona, Spain \\ ${ }^{\mathrm{g}}$ CIBER de Fragilidad y Envejecimiento Saludable (CIBERFES), Instituto de Salud Carlos III, Madrid, Spain
}

\begin{abstract}
We explored the impact of the Spanish COVID-19 strict home confinement on mental health and cognition in non-infected subjects $(\mathrm{N}=16,60-80$ years) diagnosed with subjective cognitive decline and $A P O E \varepsilon 3 / \varepsilon 4$ carriers. Mental health was monitored for 2 months on a daily, weekly, or monthly basis, and compared to pre-confinement values. Emotional distress, anxiety, and depression scores increased to pathological threshold values during and after confinement. Those with lower mood during confinement experienced a decline in their mood after confinement. Cognition did not change. These preliminary results suggest that mental health consequences of corona measures in preclinical stages of Alzheimer's disease should be further evaluated.
\end{abstract}

Keywords: Alzheimer's disease, confinement, COVID-19, mental health, subjective cognitive decline

\footnotetext{
${ }^{1}$ PENSA Study Group. From IMIM: Rafael de la Torre, Laura Forcano, Neus Pizarro, Albert Puig-Pijoan, Natalia SoldevilaDomenech, Anna Boronat, Thais Lorenzo, Aida Cuenca, Julian Mateus, Iris Piera, Ana Aldea, Patricia Diaz-Pellicer, Mara Dierssen, Maria Gomis. From BBRC: Jose Luis Molinuevo, Iva Knezevic, Sofia Menezes-Cabral, Anna Soteras, José Maria González-de-Echávarri, Gonzalo Sánchez-Benavides, Juan Domingo Gispert, Karine Fauria, Carolina Minguillón.

*Correspondence to: Rafael de la Torre Fornell, Integrative Pharmacology and Systems Neurosciences Research Group, Neu-
}

\section{INTRODUCTION}

The current COVID-19 pandemic has involved social and physical distancing as well as specific situations for individuals such as social isolation,

rosciences Research Program, Hospital del Mar Medical Research Institute (IMIM), Carrer Doctor Aiguader, 88, PRBB building, 08003 Barcelona, Spain. Tel.: +34 933160 484; E-mail: rtorre@imim.es. 
unemployment, and financial difficulties which may have consequences on mental health and mood [1, 2]. Quarantines from previous pandemics have been linked to negative psychological effects that can be long lasting, particularly in vulnerable groups such as older adults [3]. Understanding the impact of drastic non-therapeutic measures such as strict home confinement on mental health is necessary as further periods of physical distancing are likely to occur [4].

Subjective cognitive decline (SCD) is considered a preclinical stage of Alzheimer's disease and relateddementias where individuals experience a decline in cognition while performance on cognitive tests is normal [5]. Individuals with SCD are recognized as the optimal target population for dementia prevention trials [6]. Recent cross-sectional studies in Italian and Dutch population suggest that COVID-19 public health measures, including social distancing and lockdown, could cause an imbalance in the lifestyle and mental health of individuals with SCD that could persist once such measures are withdrawn $[7,8]$.

We were interested in exploring the impact of the strict Spanish COVID-19 home confinement (decreed from March to May 2020) in mental health, mood, and cognition in SARS-CoV-2 noninfected older adults at increased risk of developing Alzheimer's disease (Fig. 1). Changes in physical activity, sleep duration, and anthropometry were also evaluated. This report concerns a group of individuals meeting SCD criteria that were the object of an intensive follow-up during two months of confinement and post-confinement periods.

\section{METHODS}

We carried out an observational study with 16 individuals ( 10 women and 6 men) aged $60-80$ years with at least 4 SCD-Plus features [5] and $A P O E \varepsilon 4$ carriers (all were $A P O E \varepsilon 3 / \varepsilon 4$ ). All reported absence of COVID-19, they were tested for SARS-CoV-2 after confinement, and none presented antibodies detected by a chemiluminescence-based test.

All participants were recruited in the context of the PENSA multimodal intervention clinical trial [9]. The inclusion and exclusion criteria of the PENSA Study can be found at ClinicalTrials.gov (Identifier: NCT03978052). As part of the baseline assessments for the PENSA study, subjects were evaluated in the clinic for mental health, cognition, and anthropometry in the months previous to the pandemic, but none of them had initiated the intervention at the time of the COVID-19 outbreak.

Subjects were explored for emotional distress and possible mental health disorders with the General Health Questionnaire (GHQ-28) [10], administered before, during, and after the home confinement decreed by Spanish authorities. Anxiety

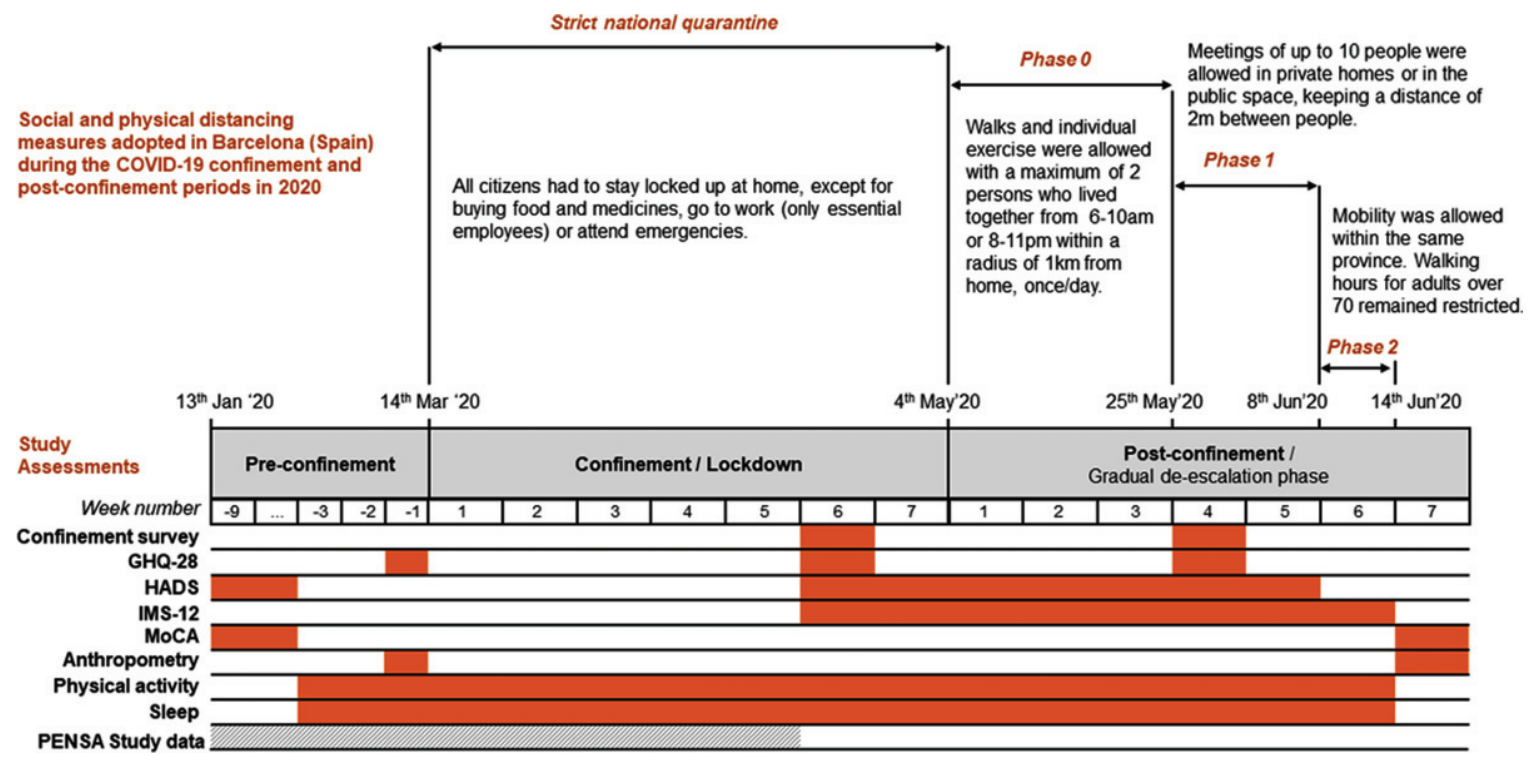

Fig. 1. Chronology of social and physical distancing measures adopted in Barcelona (Spain) during the COVID-19 confinement and postconfinement periods in 2020, together with the schedule of study assessments. GHQ-28, General Health Questionnaire; HADS, Hospital Anxiety and Depression Scale; IMS-12, Immediate Mood Scaler; MoCA, Montreal Cognitive Assessment test. 
and depression were weekly evaluated with the Hospital Anxiety and Depression Scale (HADS) [11], and mood was daily evaluated with the Immediate Mood Scaler (IMS), a measure capable of capturing the within-subject variability in mood states over time [12]. An adapted version of the COVID19 confinement survey [13] was also administered during and after confinement. This survey and the previous mental health questionnaires (GHQ-28, HADs, IMS) were administered by email during and after confinement. Cognitive function was evaluated in the clinic by qualified neuropsychologists with the Montreal Cognitive Assessment Test (MoCA) pre- and post-confinement [14]. Changes in anthropometry were measured in the clinic by bioimpedance with a TANITA system (Tanita Corp., Tokyo, Japan). Finally, the daily number of steps and hours of sleep were obtained from a subsample of 6 participants $(2 \mathrm{men})$ that wore a wristband activity tracker (Model: Fitbit ${ }^{\circledR}$ Charge 3: Fitbit Inc, San Francisco, CA) almost uninterruptedly for 17 weeks before, during, and after home confinement.

We obtained descriptive statistics of study variables across measurement occasions, including absolute and relative frequencies (\%) for categorical variables, and mean and standard deviation (SD) or 95\% confidence intervals (CI95\%) for numeric variables. Score differences in GHQ-28 and HADs over time and pre/post changes in cognition and anthropometrics were computed using linear mixed effects models, which are appropriated for longitudinal data with limited sample size [15]. Tukey's HSD (honestly significant difference) test was used to carry out post-hoc pairwise comparisons. The intra-class correlation coefficient (ICC) was computed to assess the within-person variance in mood and sleep duration, as it represents the between-person variance relative to total variance. Finally, a description of mood trajectories over time was performed using linear latent class mixed models [16], so that individuals were classified into distinct groups based on their response patterns in the IMS-12. Best model fit was selected among a finite set of models, including up to three classes based on lower Bayesian Information Criteria (BIC), a minimum of three respondents per class and distinction between classes. Statistical analyses were performed with R (Version 3.6.0, R Foundation for Statistical Computing, Vienna, Austria). Significance was set as $p<0.05$.

The study protocol was approved by the Drug Research Ethical Committee of the Parc de Salut
Mar (ID Number: IMIM/PENSA) and the informed consent was obtained from all participants.

\section{RESULTS}

There were 10 women $(62.5 \%)$ and the mean age was $65.8(\mathrm{SD}=3.75)$ years. Most participants were retired $(75.0 \%)$ and married $(75.0 \%)$, only one participant lived alone, and all reported a medium socioeconomic status. The most prevalent SCD-Plus criteria were persistence of SCD over time (93.8\%), memory-centered subjective decline $(87.5 \%)$ and seek of medical help (87.5\%). $20 \%$ of participants fulfilled all the 7 SCD-Plus criteria [5]. See Supplementary Table 1 for a detailed description of the study population.

During and after confinement, participants were found to be concerned about suffering from COVID19 , suffering from an emergency and not receiving any care, or concerned about the future. $43.8 \%$ had a relative or close friend whose cause of death was diagnosed as COVID-19. Only one participant reported poor social support, and the majority frequently communicated with family and friends. Most reported no changes regarding their consumption of fruits, vegetables, legumes, sugary drinks, alcohol, tobacco and tranquilizers, sedatives or sleeping pills. Anthropometric measures (body weight, abdominal girth, and percentage of free fat mass, muscle mass, bone mass, and fat mass) did not change after confinement $(p>0.05)$. The number of daily steps greatly decreased from a mean of 11,518 (CI95\% $10,667-12,370)$ in the pre-confinement period to 6,944 (CI95\% 6,606-7,283) in the confinement period and recovered their mean levels after confinement reaching 12,621 steps/day (CI95\% 12,059, 13,183) (Fig. 2A). Finally, during confinement, sleep duration presented higher intra-individual variability (ICC $=5.4 \%$, CI95\% $1.0-30.5 \%)$ than before (ICC $=20.6 \%$, CI95\% 4.7-72.1\%) and after confinement (ICC $=32.6 \%$, CI95\% 14.5-75.2\%) (Fig. 2B).

Perceived mental health worsened during confinement and this persisted in the post-confinement period (Fig. 3). Before confinement, only one participant was classified with a probable mental health disorder (scoring $\geq 6$ points in the GHQ-28), while after confinement all scored within the range of a probable mental health disorder (Fig. 3A). The mean GHQ-score before confinement was $1.1(\mathrm{SD}=2.3)$ points, it increased to $8.2(\mathrm{SD}=3.2)$ points during confinement and finally fell to $7.0(\mathrm{SD}=0.8)$ points 

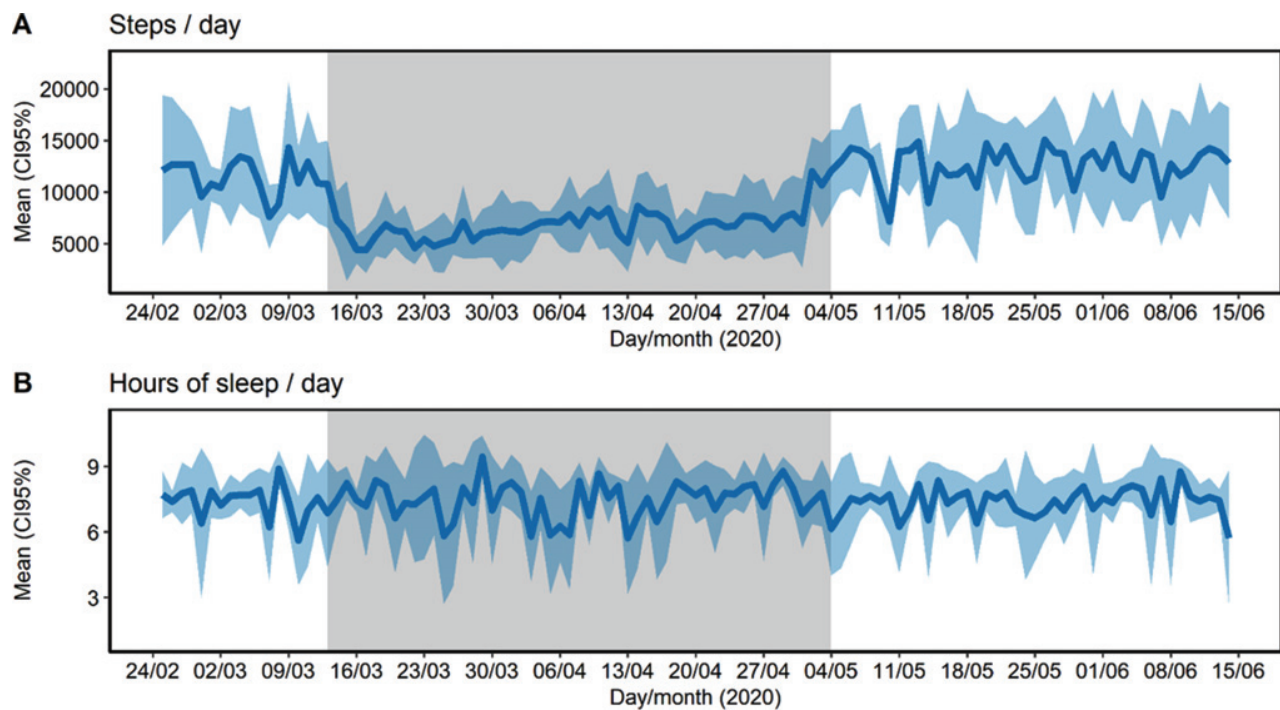

Fig. 2. Steps (A) and sleep duration (B) collected uninterruptedly during 16 weeks of 2020 of pre-confinement (17 days), confinement (53 days), and post-confinement period (42 days). Physical activity and sleep data were obtained with an activity tracker (model: Fitbit Charge 3 (C). Grey area represents the confinement period.

after confinement $(p<0.001)$. Social dysfunction was the main aspect affected by confinement $(p<0.001)$. The chronic GHQ score (cGHQ) did not change during the studied period ( $p=0.977)$, indicating the absence of chronic or persistent mental health problems.

As shown in Fig. 3B, the HADs total score increased during the confinement and post-confinement period, compared to the preconfinement assessment $(p<0.001)$. Moreover, more than half of participants fluctuated in the area of moderate or probable emotional disorder during the confinement and post-confinement period, while before confinement only 5 participants met such criteria. The mean HADs anxiety score did not change during the study period $(p=0.298)$. Conversely, the HADs depression score increased during the confinement period $(p<0.001)$, with a mean increase of 2.5 points (CI95\% 1.1, 4.0) between the pre-confinement period and confinement week 6 (Supplementary Figure 1).

Mood presented high intra-individual variability $(\mathrm{ICC}=19.3 \%$, CI95\% 10.7-37.6\%). Three different latent mood trajectories were identified and were labeled as high $(\mathrm{N}=7 ; 43.75 \%$ of individuals), average $(\mathrm{N}=6 ; 37.5 \%)$, and low $\operatorname{mood}(\mathrm{N}=3 ; 18.75 \%)$ (Fig. 3C). Individuals with low mood presented a decrease of 8.5 points (CI95\% -3.7, -13.3) in the post-confinement week $1(p<0.001)$, compared to the confinement week 6 , and lower mood levels remained during all the post-confinement period $(p<0.001)$. Individuals with average mood presented a relatively stable mean mood status. Finally, individuals with high mood presented a slight increase in mood from post-confinement week $3(p<0.01)$.

Regarding cognition, MoCA total score $(\mathrm{N}=11)$ in the pre-confinement period (25.6 points, $\mathrm{SD}=2.3$ ) did not change after confinement $(26.8, \mathrm{SD}=2.6$; $p=0.669)$. As expected, some practice effects were observed in the cognitive performance of some individuals since the time interval between the pre- and post-evaluations was less than 6 months [17]. However, after confinement a 3-point decrease in global cognition (MoCA total score) was observed in 18.2\% of participants and $63 \%$ scored lower in one or more cognitive domains, including language, abstraction, orientation, and attention.

\section{DISCUSSION}

In this report, we longitudinally and intensively evaluate how a strict confinement such as the one decreed in Spain during the beginning of the COVID-19 pandemic may influence mental health perception and experienced levels of anxiety, depression, and mood in non-infected subjects meeting SCD-plus criteria and carrying the $A P O E \varepsilon 4$ genetic marker for Alzheimer's disease. Our results, although merely exploratory and obtained in a limited sample, 
A GHQ-28 score

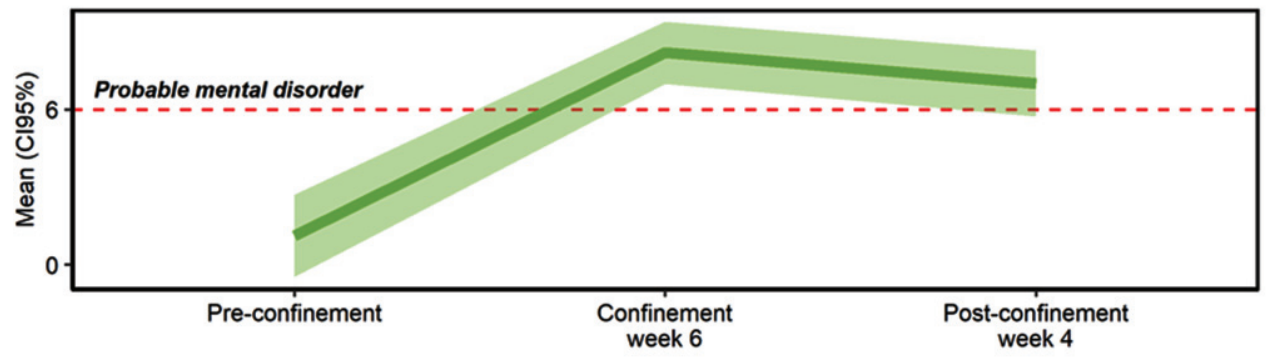

B HADs total score

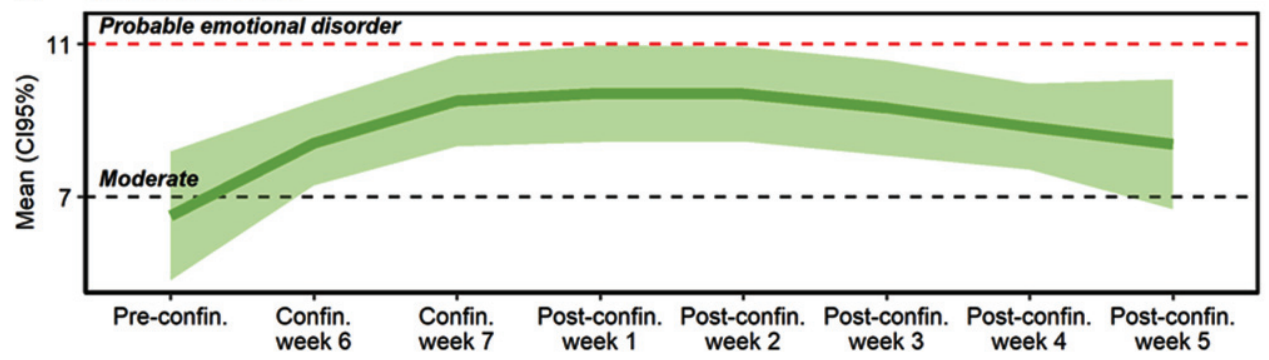

C IMS-12 total score

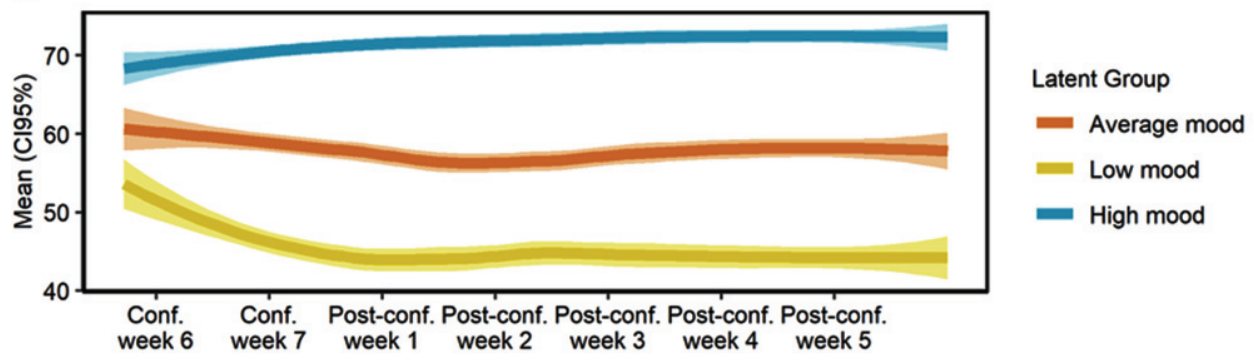

Fig. 3. Evolution of mental health before, during and after the confinement. A) General Health Questionnaire (GHQ-18) total score. B) Hospital Anxiety and Depression Scale (HADs) total score. C) Immediate Mood Scaler (IMS-12) total score. Conf. or confin, confinement; Pre-confin, pre-confinement; Post-confin, post-confinement.

highlight that the indirect impact of social distancing measures on the mental health of individuals at increased risk of Alzheimer's disease could have long-term consequences. Thus, these preliminary results should be evaluated more in depth in future studies, especially in larger cohorts of individuals with SCD, but also in the elderly population at large.

Subjects report a worsening perception of mental health that does not fully recover until weeks after the end of confinement. All scores measured by the GHQ-28 were slightly altered by confinement. In fact, all subjects during and after confinement displayed global GHQ-28 scores above pathological threshold values. Any change in the global GHQ-28 score essentially relies on the social dysfunction score which is particularly understandable within the context of the current pandemic and confinement measures. The HADs scale used to evaluate depression and anxiety shows that during confinement there was a mild increase in the depressive symptomatology experienced by subjects. Using IMS and latent variables statistical analysis, we were able to group subjects following mood trajectories throughout the study period. It appears that those subjects with the highest scores during confinement experienced little changes in their mood, while those with lower scores show worse overall scores after confinement. Therefore, globally the confinement had a deleterious effect on mental health and mood in our group of subjects, 
in accordance with previous studies in older adults and SCD individuals.

Results from the MoCA instrument used to screen cognitive performance suggest that changes, if any, were very mild. This instrument is not the most adequate for evaluating in-depth cognitive functioning but was available in the framework of this study. Other limitations of the present study include the fact that, due to the small sample size, analyses were not adjusted for potential confounders (gender, age, educational level [4]) and mood trajectories included latent groups composed of only 3 participants. Given that anxiety and depressive symptoms frequently cooccur with SCD [18], all these limitations should be contemplated when considering the generalizability of our results and when designing future studies.

As stated earlier, study participants were recruited in a clinical trial involving a multimodal intervention in lifestyle habits for the prevention of cognitive decline in subjects meeting SCD-Plus criteria and carrying the $A P O E \varepsilon 4$ genetic marker (PENSA Study) [9]. Participation was halted due to the COVID-19 pandemic. However, the research team maintained contact with participants during and after confinement. Data from subjects was analyzed as pooled allowing comparison between mean scores before, during, and after confinement. However, results from this group of subjects show that individual trajectories are in many instances quite different and any intervention should take this into account. This is also true for data analysis where approaches like $\mathrm{N}$-of- 1 clinical trials allow for individual data analysis (although further analysis of aggregated data is also possible) [19]. In this context, some of the tests we used were administered weekly or monthly, whereby subjects were requested to recall their behavior or health perception during this time interval, preventing a more repeated administration. For other variables like mood, data is collected daily and in the case of sleep quality and physical activity, data is collected continuously. This amount of data should suffice to describe individual trajectories and understand efficacy or non-response to clinical interventions. These findings are informative for precision prevention trials and therapies.

\section{ACKNOWLEDGMENTS}

This project is supported by the Alzheimer's Association (18PTC-R-592192) and by Instituto de Salud Carlos III (ISCIII PI17/00223, co-funded by the European Regional Development funds). NSD was recipient of a predoctoral fellowship \#2019-DI47 from the DIUE-AGAUR of the "Generalitat de Catalunya". The funders had no role in study design; in the collection, analysis and interpretation of the data; in the writing of the report and in the decision to submit the article for publication. The Physiopathology of Obesity and Nutrition Networking Biomedical Research Center (CIBEROBN) is an initiative of Instituto de Salud Carlos III (ISCIII), Madrid, Spain.

Authors' disclosures available online (https:// www.j-alz.com/manuscript-disclosures/20-1408r1).

\section{SUPPLEMENTARY MATERIAL}

The supplementary material is available in the electronic version of this article: https://dx.doi.org/ 10.3233/JAD-201408.

\section{REFERENCES}

[1] Centers for Disease Control and Prevention. Mental Health and Coping During COVID-19.

[2] United Nations (2020) Policy Brief: COVID-19 and the Need for Action on Mental Health. World Health Organization, Geneva, Swithzerland.

[3] Brooks SK, Webster RK, Smith LE, Woodland L, Wessely S, Greenberg N, Rubin GJ (2020) The psychological impact of quarantine and how to reduce it: Rapid review of the evidence. Lancet 395, 912-920.

[4] Niedzwiedz CL, Green M, Benzeval M, Campbell D, Craig P, Demou E, Leyland AH, Pearce A, Thomson RM, Whitley E, Katikireddi SV (2020) Mental health and health behaviours before and during the COVID-19 lockdown: Longitudinal analyses of the UK Household Longitudinal Study. J Epidemiol Community Heal, doi: 10.1136/jech2020-215060

[5] Jessen F, Amariglio RE, Buckley RF, van der Flier WM, Han Y, Molinuevo JL, Rabin L, Rentz DM, Rodriguez-Gomez O, Saykin AJ, Sikkes SAM, Smart CM, Wolfsgruber S, Wagner M (2020) The characterisation of subjective cognitive decline. Lancet Neurol 19, 271-278.

[6] Cheng Y, Chen T, Chiu M (2017) From mild cognitive impairment to subjective cognitive decline: Conceptual and methodological evolution. Neuropsychiatr Dis Treat 13, 491-498.

[7] van Maurik IS, Bakker ED, van den Buuse S, Gillissen F, van de Beek M, Lemstra E, Mank A, van den Bosch KA, van Leeuwenstijn M, Bouwman FH, Scheltens P, van der Flier WM (2020) Psychosocial effects of corona measures on patients with dementia, mild cognitive impairment and subjective cognitive decline. Front Psychiatry 11, 1-8.

[8] Di Santo SG, Franchini F, Filiputti B, Martone A, Sannino S (2020) The effects of COVID-19 and quarantine measures on the lifestyles and mental health of people over 60 at increased risk of dementia. Front Psychiatry 11, 578628.

[9] Kivipelto M, Mangialasche F, Snyder HM, Allegri R, Andrieu S, Arai H, Baker L, Belleville S, Brodaty H, Brucki SM, Calandri I, Caramelli P, Chen C, Chertkow H, Chew E, Choi SH, Chowdhary N, Crivelli L, De La Torre R, Du Y, Dua T, Espeland M, Feldman HH, Hartmanis M, 
Hartmann T, Heffernan M, Henry CJ, Hong CH, Håkansson $\mathrm{K}$, Iwatsubo T, Jeong JH, Jimenez-Maggiora G, Koo EH, Launer LJ, Lehtisalo J, Lopera F, Martínez-Lage P, Martins R, Middleton L, Molinuevo JL, Montero-Odasso M, Moon SY, Morales-Pérez K, Nitrini R, Nygaard HB, Park YK, Peltonen M, Qiu C, Quiroz YT, Raman R, Rao N, Ravindranath V, Rosenberg A, Sakurai T, Salinas RM, Scheltens P, Sevlever G, Soininen H, Sosa AL, Suemoto CK, TaintaCuezva M, Velilla L, Wang Y, Whitmer R, Xu X, Bain LJ, Solomon A, Ngandu T, Carrillo MC (2020) World-Wide FINGERS Network: A global approach to risk reduction and prevention of dementia. Alzheimers Dement 16, 1078-1094.

[10] Goldberg DP, Gater R, Sartorius N, Ustun TB, Piccinelli M, Gureje O, Rutter C (1997) The validity of two versions of the GHQ in the WHO study of mental illness in general health care. Psychol Med 27, 191-197.

[11] Zigmond AS, Snaith RP (1983) The Hospital Anxiety and Depression Scale. Acta Psychiatr Scand 67, 361-370.

[12] Nahum M, Van Vleet TM, Sohal VS, Mirzabekov JJ, Rao VR, Wallace DL, Lee MB, Dawes H, Stark-Inbar A, Jordan JT, Biagianti B, Merzenich M, Chang EF (2017) Immediate Mood Scaler: Tracking symptoms of depression and anxiety using a novel mobile mood scale. JMIR mHealth uHealth $\mathbf{5}$, e44.

[13] Departament de Salut, Generalitat de Catalunya. Cuestionario de salud en tiempos de confinamiento por el coronavirus.
[14] Ojeda N, Del Pino R, Ibarretxe-Bilbao N, Schretlen DJ, Pena J (2016) Montreal Cognitive Assessment Test: Normalization and standardization for Spanish population. Rev Neurol 63, 488-496.

[15] Muth C, Bales KL, Hinde K, Maninger N, Mendoza SP, Ferrer E (2016) Alternative models for small samples in psychological research: Applying linear mixed effects models and generalized estimating equations to repeated measures data. Educ Psychol Meas 76, 64-87.

[16] Ram N, Grimm KJ (2009) Methods and Measures: Growth mixture modeling: A method for identifying differences in longitudinal change among unobserved groups. Int J Behav Dev 33, 565-576.

[17] Cooley SA, Heaps JM, Bolzenius JD, Salminen LE, Baker LM, Scott SE, Paul RH (2015) Longitudinal change in performance on the montreal cognitive assessment in older adults. Clin Neuropsychol 29, 824-835.

[18] Hill NL, Mogle J, Wion R, Munoz E, DePasquale N, Yevchak AM, Parisi JM (2016) Subjective cognitive impairment and affective symptoms: A systematic review. Gerontologist 56, e109-e127.

[19] Soldevila-Domenech N, Boronat A, Langohr K, de la Torre $\mathrm{R}$ (2019) N-of-1 clinical trials in nutritional interventions directed at improving cognitive function. Front Nutr 6, 110. 